Article

\title{
Optimal Microgrid Topology Design and Siting of Distributed Generation Sources Using a Multi-Objective Substrate Layer Coral Reefs Optimization Algorithm
}

\author{
Silvia Jiménez-Fernández ${ }^{1, *(1)}$, Carlos Camacho-Gómez ${ }^{1}$, Ricardo Mallol-Poyato ${ }^{1}$, \\ Juan Carlos Fernández ${ }^{2}{ }^{\circledR}$, Javier Del Ser ${ }^{3}$, Antonio Portilla-Figueras ${ }^{1}$ and Sancho Salcedo-Sanz ${ }^{1}$ \\ 1 Department of Signal Processing and Communications, Universidad de Alcalá, 28805 Alcalá de Henares, \\ Madrid, Spain; carlos.camacho@uah.es (C.C.-G.); ricardo.mallol@uah.es (R.M.-P.); \\ antonio.portilla@uah.es (A.P.-F.); sancho.salcedo@uah.es (S.S.-S.) \\ 2 Department of Computer Science and Numerical Analysis, Universidad de Córdoba, 14701 Córdoba, Spain; \\ jfcaballero@uco.es \\ 3 TECNALIA, 48160 Derio, Bizkaia, Spain; javier.delser@tecnalia.com \\ * Correspondence: silvia.jimenez@uah.es; Tel.: +34-91-885-6648
}

Received: 4 December 2018; Accepted: 21 December 2018; Published: 30 December 2018

\begin{abstract}
In this work, a problem of optimal placement of renewable generation and topology design for a Microgrid (MG) is tackled. The problem consists of determining the MG nodes where renewable energy generators must be optimally located and also the optimization of the MG topology design, i.e., deciding which nodes should be connected and deciding the lines' optimal cross-sectional areas (CSA). For this purpose, a multi-objective optimization with two conflicting objectives has been used, utilizing the cost of the lines, $C$, higher as the lines' CSA increases, and the MG energy losses, E, lower as the lines' CSA increases. To characterize generators and loads connected to the nodes, on-site monitored annual energy generation and consumption profiles have been considered. Optimization has been carried out by using a novel multi-objective algorithm, the Multi-objective Substrate Layers Coral Reefs Optimization algorithm (Mo-SL-CRO). The performance of the proposed approach has been tested in a realistic simulation of a MG with 12 nodes, considering photovoltaic generators and micro-wind turbines as renewable energy generators, as well as the consumption loads from different commercial and industrial sites. We show that the proposed Mo-SL-CRO is able to solve the problem providing good solutions, better than other well-known multi-objective optimization techniques, such as NSGA-II or multi-objective Harmony Search algorithm.
\end{abstract}

Keywords: Microgrids; network topology; distributed renewable generation; Multi-objective Substrate Layers Coral Reefs Optimization algorithm (Mo-SL-CRO)

\section{Introduction}

Local and distributed generation is on the rise due to the constant evolution and improvement of technologies related to the production of electricity using renewable energies, the storage in batteries or other energy storage systems and the intelligent management systems. These advances are leading to the development of Microgrids (MGs), small-sized networks of electricity users (loads) with local sources of electricity supply and, sometimes, even containing energy storage systems [1]. These MGs can operate connected to the main national grid or in islanded mode [2]. MGs promote installation of Distributed Generation (DG) for self-consumption [3], and a reduction of electricity transportation lines losses and, therefore, research efforts are focusing on the design and optimization of MGs' topologies, planning, energy management, etc. 
However, due to DG and the intermittent nature of renewable energy sources, design and management of MGs pose great challenges. Thus, different reviews on the planning of a MG and its optimization [4-6] show the complex procedures to consider that can be tackled as a sequence of optimization processes to obtain best results. Issues optimized vary, mainly, from the design determining the optimal power generation mix selection (renewable, diesel, etc.) to satisfy demand for a particular area [7-9], the sizing and siting problem [10-16] or the scheduling to minimize operational costs, environmental impact, quality, etc. while covering the demand [17-20].

Focusing on DG allocation and sizing, a complex problem with non-linear objective function or constraints, heuristic algorithms represent an interesting option to solve it. A wide range of objectives are suggested in the literature to perform the siting, such as power loss reduction, stability voltage improvement, reliability improvement, cost reduction, etc. [10-24]. Normally, the few times these works refer to the loads description, they are assumed invariant or of constant power, although optimality found in these cases may not be of application when real time-varying loads are used. Moreover, a wide range of optimization techniques has been proposed as well, such as Radial Basis Functions [16], Invasive Weed Optimization [22], Plant Growth Simulation algorithm [25], Bacterial Foraging Optimization algorithm [14,26], Honey bee mating [27], Coral reefs optimization [28], etc. In all cases, optimal sizing and siting of DG are considered and effectiveness of the optimization techniques is usually tested on standard network topologies such as IEEE 33-bus [22,23,25], 69-bus [16,23] or 69-radial [22]. In all these cases, several objective functions are considered as a single one by using a weighted sum of them. In [23], the optimal sizing and siting of DG in appropriate lines in the system is performed by minimizing the power losses and operating cost using a weighted sum approach of them. No information is provided regarding the nature of the loads used.

On the contrary, another approach is to find optimal locations and sizes by optimizing several objective functions simultaneously, based on Pareto optimal fronts that yield to non-dominated solutions $[15,24,29,30]$. Sheng et al. [15] propose the use of an improved version of the well-known non-dominated sorting genetic algorithm II (iNSGA-II) to solve the multi-objective optimization with consideration of line loss, voltage regulation and voltage stability margin. Test scenario used is IEEE 33-bus and no information is provided regarding the loads used or their profiles.

In [24], the authors use power losses and voltage regulation as objectives, and an improved Multi-objective Harmony Search optimization technique based on Pareto fronts. Regarding the loads used, two prototype systems are considered, but further information is not provided.

In [29] a strategy to place DG units in uncertain environments (modelled using fuzzy numbers) is presented. Objective functions to be minimized are monetary cost (that includes investment, operation cost and cost of losses), technical risks (including voltage and loading constraints violation) and economic risk due to uncertainty of electricity's market price.

Optimization problems feature two main stages. First, choosing the objectives and constraints, as well as the generation and loads' power profiles. Later, choosing the optimization algorithm to determine the optimal solution.

In this work the type of microgrid considered is formed by several electric points of consumption (loads), each located at a different "node". The MG's nodes are connected conforming a topology, that may be a radial topology, where all nodes are connected to a main one (the PCC, Point of Common Coupling, the node connecting to the main grid), a fully mesh topology, where all nodes are connected to each other, or any in-between topology. To determine the design criteria, several options referred in the literature by different authors have been presented before. In this work we have chosen to consider the MG's economic cost and the energy losses associated to the topology, as most authors are prone to consider them as key design criteria. In addition, siting of DG is added to the problem, increasing the difficulty of the design process. Furthermore, instead of the use of constant power loads attached to the nodes, on-site monitored annual industrial and commercial consumption profiles are considered. Eventually, optimal siting of DG and optimal topology design (determining which 
nodes should be connected by lines and which cross-sectional areas should be used) will be analized considering the MG's costs and its energy losses. Moreover, these aspects are also conflicting to one another: the more expensive the topology is (higher lines' cross-sectional areas), the lower the energy losses are. Therefore, we propose the use of a multi-objective optimization approach to determine a front of non-dominated solutions (no solution in the front is better or worse than the others, and the decision maker needs to choose the most fitted solution for his/her interests). In this paper we propose modifying the Coral Reefs Optimization (CRO) algorithm [31], an evolutionary-based algorithm that shows good convergence and optimization skills, to be able to tackle multi-objective approaches. To do so, we propose to enhance the algorithm with Substrate Layers [32] and then, adapt it to be able to generate a full Pareto front to tackle conflicting objectives. In conclusion, the main contribution of this work is presenting the novel and powerful algorithm "Multi-objective Substrate Layers Coral Reefs Optimization algorithm (Mo-SL-CRO)", and testing it on a MG equipped with several loads (represented by real monitored consumption profiles) where distributed renewable generation needs to be allocated.

The remainder of the paper is organized as follows: Section 2 states the formulation of the problem at hand, defining all variables considered and the mathematical formulation of the objective functions. Section 3 explains the methodology used by introducing the novel multi-objective Substrate Layer Coral Reefs Optimization algorithm, the different substrates considered, solutions' encoding and metrics to analyze the algorithm's performance. Finally, results obtained are given in Section 4 and some conclusions are drawn in Section 5.

\section{Problem Formulation}

The aim of this work is to optimize the network topology and structure of a microgrid, considering the design of the lines that connect the nodes in the grid (which nodes should be interconnected and which should not, and which cross-sectional area (CSA) should be used for those lines) and the best node(s) to locate each DG unit. This optimization will consider simultaneously two conflicting to one another objectives: lines' deployment cost, $C$, higher as the line's CSA increases, vs the energy losses, $E$, lower as the line's CSA increases. However, any other objectives can be added to the problem to be optimized by the proposed algorithm.

Let us consider a MG that formed by $N$ nodes, where the power lines that connect nodes $i$ and $k$ in the grid may have different cross-sectional areas represented by $a_{i k}$. Therefore, an $N \times N$ matrix, $\mathcal{S}$, can be formed to represent the microgrid's topology, where each position $\mathcal{S}(i, k)$ stands for the CSA of the line connecting nodes $i$ and $k$. If those nodes are not connected, and there is no power line between them, a zero will be used at position $(i, k)$. Note that, by definition, as a node cannot be connected to itself by any line, $\forall i \in\{1, \ldots, N\}, \mathcal{S}(i, i)=0$.

Furthermore, a given number of different types of loads (i.e., industrial, commercial or domestic) are allocated at the nodes of the MG and are characterized, in this work, by their annual power hourly-consumption profiles. Other elements of the microgrid to be considered are renewable energy generators (specifically, wind-turbines and photovoltaic generators have been used) that may be placed conveniently at any node $N$. Thus, an $N \times 2$ matrix $\mathcal{T}$ that represents the renewable energy generators to be allocated at each node $N$ can be defined.

Consequently, the objective of this work is to optimize the topology, $\mathcal{S}$, and assign the generators, $\mathcal{T}$, using two design criteria: the total energy losses of the lines, $E$, and the total cost of those lines, $C$.

To obtain the energy losses of a given topology (where the lines' CSAs and the location of generators and consumption loads are set), the power flowing over the lines of the grid has to be determined. This procedure is iterative [33] and time consuming, thus, under certain circumstances (such as those ocurring in small-sized MGs with low-voltage supply networks), several assumptions can be made that simplify the process to obtain the power losses [34]. Let us define $\mathcal{L}_{i k}$ as the power losses corresponding to the line connecting the pair of nodes $(i, k)$ (Equation (1)). These losses are 
determined by the power transmitted over that line at time $t, P_{i k}^{t}$, obtained using the DC power flow method [34].

$$
\mathcal{L}_{i k}^{t}=\left(\frac{P_{i k}^{t}}{V_{\text {nom }}}\right)^{2} \cdot R_{i k}=\left(\frac{P_{i k}^{t}}{V_{\text {nom }}}\right)^{2} \cdot \rho \cdot \frac{l_{i k}}{a_{i k}}
$$

where $R_{i k}$ is the resistance of the line that connects the pair of nodes $(i, k)$. The resistance is characterized by the cross-sectional area $\left(a_{i k}\right)$, the length $\left(l_{i k}\right)$, and the resistivity of the material $(\rho)$. $V_{\text {nom }}$ is a constant standing for the nominal voltage value (Volts) of the MG. Consequently, the line's energy losses, $\mathcal{E}_{i k}$, are obtained as depicted in Equation (2).

$$
\mathcal{E}_{i k}=\sum_{t=1}^{T} \mathcal{L}_{i k}^{t}
$$

Finally, summing up each line's energy losses, the total energy losses are obtained (Equation (3)).

$$
E(\mathbf{x})=\sum_{i=1}^{N-1} \sum_{k=i+1}^{N} \mathcal{E}_{i k}
$$

where $\mathbf{x}$ stands for the individual that represents a particular MG network topology $(\mathcal{S})$ and a specific generators' location $(\mathcal{T})$.

Additionally, the location of the generators yields to different topologies that present certain infrastructure costs that have been approximated as in Equation (4).

$$
C(\mathbf{x})=\sum_{i=1}^{N} \sum_{k=i}^{N} l_{i k} \cdot\left[C_{i n s}+a_{i k} \cdot C_{m a t}\right]
$$

Note that $C_{m a t}$ stands for the unity cost per $m \cdot \mathrm{mm}^{2}$ of the line and $C_{i n s}$ stands for the unity cost per $m$ of the lines' installation.

\section{Materials and Methods}

This Section starts specifying the algorithm proposed to solve the multi-objective optimization problem. It then continues with the definition of the substrates chosen to empower the algorithm in different manners: generation of best larva candidates, stretching of the Pareto front, etc. Next, a subsection determining the individual's encoding is provided. Then, the flowchart of the overall procedure is presented, showing the Mo-SL-CRO steps and how the individual's fitness (health) functions are obtained using Equations (3) and (4), introduced in Section 2. Finally, the optimization performance metrics needed to objectively compare Pareto optimal fronts is introduced, as each solution is not better or worse than the others contained in the front, and it is the decision maker the one that has to decide which solution suits best for his/her interests.

\subsection{The Multi-Objective Substrate Layers Coral Reefs Optimization Algorithm}

The original Coral Reefs Optimization (CRO) Algorithm [31,35,36] is an evolutionary-based algorithm that simulates the processes occurring in a coral reef. Furthermore, when substrate layers (each representing a different exploration mechanism) are implemented constituting the so called Substrate Layers Coral Reefs Optimization algorithm (SL-CRO), survival of coral larvae depending on the substrate is emulated, and competitive co-evolution is added to the the original CRO algorithm [32,37].

Nevertheless, this is a mono-objective algorithm and if the problem under study needs to optimize conflicting to one another objective functions, it has to be addressed as multi-objective and a new algorithm needs to be defined in order to produce a front of non-dominated solutions. In this case, a possible solution vector $\mathbf{x}_{1}$ is said to dominate another solution vector $\mathbf{x}_{2}$ (denoted by $\mathbf{x}_{1}>\mathbf{x}_{2}$ ) if and 
only if $\mathbf{x}_{1}$ is not worse than $\mathbf{x}_{2}$ in all $i$ objectives $\left(\forall i: f_{i}\left(\mathbf{x}_{1}\right) \leq f_{i}\left(\mathbf{x}_{2}\right), i=1, \ldots, m\right)$ and is strictly better than $\mathbf{x}_{2}$ in at least one objective $\left(\exists i \mid f_{i}\left(\mathbf{x}_{1}\right)<f_{i}\left(\mathbf{x}_{2}\right), i=1, \ldots, m\right)$.

This subsection explains the multi-objective non-domination based Substrate Layers Coral Reefs Optimization algorithm (Mo-SL-CRO). Let $\mathcal{R}$ be an $R_{1} \times R_{2}$ grid simulating the reef, where each cell is represented by three values $(i, j, s) .(i, j)$ denotes the cell location in $\mathcal{R}$ and is able to allocate a coral or a colony of corals $\left(\Psi_{i, j}\right)$. Index $s \in\left\{S_{1}, \cdots, S_{N}\right\}$ represents the substrate layer associated to position $(i, j)$. Thus, the reef is divided in $S_{N}$ different substrate layers.

Each coral (solution) is labeled with two or more associated health functions $f\left(\left(\Psi_{i j}\right)^{k}\right): \mathcal{A} \rightarrow \mathbb{R}$ $\left(k \in\left\{S_{1}, \cdots, S_{N}\right\}\right)$ that correspond to the problem's opposite objective functions. The CRO is based on the fact that the reef will evolve and develop as long as healthier or stronger corals (which represent better solutions to the problem at hand) survive, while less healthy corals perish. In the multi-objective algorithm a coral is considered stronger if it dominates the existing coral in all objectives.

The algorithm starts with an initialization process, where a percentage of the elements of the grid is assigned with random corals, leaving the other elements unoccupied. These holes are available to settle new corals in later phases of the algorithm. The initial occupation factor (i.e., the rate between free and occupied cells in $\mathcal{R})$ is a parameter of the algorithm denoted as $\rho_{0}\left(0<\rho_{0}<1\right)$.

The second phase simulates the formation of the reef, implementing the processes of reproduction and prey, and is repeated $k \in\{1 \ldots K\}$ iterations. The different reproduction mechanisms available in nature are recreated by sequentially applying different operators:

1. Sexual reproduction: The $C R O$ algorithm implements three different types of sexual reproduction: external, internal and asexual.

1.1. External sexual reproduction or Broadcast spawning. In nature, most reef's corals reproduce ejecting gametes. These gametes combine and produce a new larvae. To model this procedure in the SL-CRO algorithm, a high fraction $F_{e}$ of the reef's existing corals are chosen (the broadcast spawners). For a given coral, its substrate layer $s$ is checked to determine the exploration strategy to apply (Section 3.2). Depending on the operator to be used in that particular substrate, one, two or more corals will be chosen to create the new larva. Note that if a coral is chosen to father a larva at a given iteration $k$, it will not be selected for other external reproduction purposes during that iteration. The selection of the corals can be performed using different techniques, i.e., randomly, uniformly, or using any selection approach proportionate to the health function (e.g., roulette wheel).

1.2. Internal sexual reproduction or Brooding. In nature, the reproduction technique of hermaphrodite corals is called brooding. The CRO algorithm models brooding using any type of mutation mechanism. To do so, it considers the remaining fraction of corals that were not chosen for broadcast spawning $\left(F_{i}=1-F_{e}\right)$. Next, a percentage $P_{i}$ of the coral is mutated.

1.3. Asexual reproduction or Budding. Another reproduction technique in corals is asexual, where new larvae are formed by fragmentation or budding. The CRO models this nature's mechanism using a mutation process. All corals in the reef are considered and sorted by their health function $\left(f\left(\Psi_{i j}\right)\right)$. Next, a fraction $F_{a}$ of the healthier individuals are chosen. Then, to provide more variability to the reef's population, new larvae are formed using mutation with probability $P_{a}$. 
2. Larvae settlement. Once all new larvae are formed at iteration $k$, they try to settle down and grow in the reef. Each larva will randomly attempt at setting at a position $(i, j)$ of the reef. If there is no coral at it, the coral grows there, independently of its health function. On the contrary, if the position is already occupied, the new larva will set only if it dominates in all axes the existing larva (its health functions are better than the fitness functions of the existing coral). We define a number of attempts $\mathcal{N}_{\text {att }}$ for a larva to set in the reef: after $\mathcal{N}_{\text {att }}$ unsuccessful tries, it will be discarded and die.

3. Prey. In nature, there are some corals that die during the formation of the reef. This process is simulated choosing a small amount of settled corals $\left(F_{p}\right)$ that are removed with a small probability $\left(P_{p}\right)$ at the end of each reproductive iteration $k$. Thus, some space in the reef is freed to allow for the settlement of worst-fitted larvae during next generation (iteration $k+1$ ), opening the search space to new .

To determine which corals die during this step, a procedure similar to that applied in NSGA-II [38] is used in the Mo-SL-CRO algorithm. First, a non-dominated sort of the reef's population is performed (calculating each coral's rank). Then, an estimate of the density of solutions surrounding each particular coral is obtained, using the crowded comparison operator. Finally, between two corals with differing non-domination ranks, the coral with lower rank will be kept. If both belong to the same rank, the coral located in a region with lesser number of points will be kept.

\subsection{Substrate Layers Used in This Work}

Many possible substrate layers may be used to implement the competitive co-evolution of the SL-CRO algorithm. In this work, the following substrates have been considered.

- Harmony Search (HS) [39]. It is inspired on the search of best music harmonies through improvisation. The next harmony is determined obtaining several combinations following these rules: (1) Choosing the new combination from the memory. (2) Considering a harmony from the memory that is adjacent to the given one. (3) Generating a random combination. For this purpose, two parameters are defined: Harmony Memory Considering Rate (HMCR) and Pitch Adjusting Rate (PAR) that are used to create the Harmony Search Memory (HM) needed to try new harmonies.

Considering this substrate layer means improvising a new larva using the HM, and no existing coral (parent) is needed to create it.

- Differential Evolution Mutation (DEM) [40]. Three parents (parent-A, parent-B and parent-C) are chosen randomly from the existing reef to create a new larva as follows: the weighted difference between parent- $A$ and parent- $B$ is added to parent- $C$ (weights are randomly chosen).

- 2-points Crossover (2PX). Two existing corals are chosen as parents (parent-A and parent-B) of the new larva. Then, two cross-points from parent-A are picked out randomly. New larva inherits genetic code in-between those cross-points from parent-A, and the rest from parent-B.

- Gaussian Mutation (GM). An existing coral is chosen as parent of one new larva, and a unit Gaussian distributed random value is added to each gene (element) in the coral. If it falls outside the upper or lower bounds, the new gene is adjusted to the correct bound.

- Strange attractors-based mutation (SABM). SABM [41] is a search operator that replicates fractal geometric patterns to obtain structures of non-linear dynamical systems with chaotic behaviour [42]. For this purpose, one coral made up of $T$ genes or elements $\left(\mathbf{x}=\left[x_{1} x_{2} \ldots x_{i} \ldots x_{T}\right]\right)$ is used as parent of the next generation's larva $\left(\mathbf{x}^{\prime}=\left[x_{1}^{\prime} x_{2}^{\prime} \ldots x_{i}^{\prime} \ldots x_{T}^{\prime}\right]\right)$. Each gene $x_{i}^{\prime}$ of the mutated larva is obtained as:

$$
\begin{cases}x_{i}^{\prime}=x_{i}+F v ; & \text { probability } \mathcal{P} \\ \text { or } & \\ x_{i}^{\prime}=x_{i}+F \omega ; & \text { probability }(1-\mathcal{P})\end{cases}
$$


where $\mathcal{P}$ is the probability of choosing between one expression or the other, $F$ controls the mutation width and $v$ or $\omega$ are determined using a two-dimensional quadratic map formed as:

$$
\left\{\begin{array}{l}
v=v_{\gamma}=a_{1}+a_{2} v_{\gamma-1}+a_{3} v_{\gamma-1}^{2}+a_{4} v_{\gamma-1} \omega_{\gamma-1}+a_{5} \omega_{\gamma-1}+a_{6} \omega_{\gamma-1}^{2} \\
\omega=\omega_{\gamma}=a_{7}+a_{8} v_{\gamma-1}+a_{9} v_{\gamma-1}^{2}+a_{10} v_{\gamma-1} \omega_{\gamma-1}+a_{11} \omega_{\gamma-1}+a_{12} \omega_{\gamma-1}^{2}
\end{array}\right.
$$

This procedure allows to generate very different attractors using a reduced number of parameters. In this work, the SABM has been implemented following the procedure proposed in [41] and 50 different attractors have been defined (each attractor has 12 parameters, $a_{1}-a_{12}$, associated in the range $[-1.2,1.2])$. A random attractor is used each time the operator is applied, therefore obtaining the quadratic map over a random number $\gamma \in[500,1000]$ of iterations starting from an initial condition $\left(v_{0}, \omega_{0}\right)=(0.6,0.9)$ until $v$ and $\omega$ are found. Thus, over $25^{10}$ different attractors (intermittent, convergent to a periodic orbit or chaotic) are generated.

- Simulated Binary Crossover (SBX) attempts to simulate the single-point crossover operator used in binary-encoded Genetic Algorithms, producing children (larvae) with same average value as their parents (corals) and being more likely near the parents [43]. Thus, two existing corals are chosen as parents and two different larvae are generated.

- Polynomial Mutation (PM) models the generation of offsprings using binary-encoded bit-flip mutation on real-valued decision variables, favouring mutated solutions to be near the parent generating them [44].

\subsection{Individual's Encoding}

Each solution to the problem $\mathbf{x}$, a.k.a. individual, encodes, using integer numbers, the information in $\mathcal{S}$ and $\mathcal{T}$. A first factor to be considered is that $\mathcal{S}$ is a symmetric matrix (the line connecting nodes $i$ and $k$ is the same as the one connecting $k$ and $i$ ) and the elements of the main diagonal are equal to zero (there is no line connecting a node to itself). Thus, only the upper triangular matrix without the main diagonal needs to be encoded in the individual. Notation used to refer to the CSA of the line connecting nodes $n_{1}$ and $n_{2}$ is $a_{n_{1} n_{2}}$.

The second factor is that $\mathcal{T}$ stores the number of generators per node and represents a finite quantification of the generator's size (nominal power). In this work two types of renewable energy generation have been considered: wind turbines (W) and photovoltaic generators (PV). An example of the notation used to define the number of wind turbines at node $n_{1}$ is: $N_{n_{1}}^{W}$.

Therefore, the individual is encoded as:

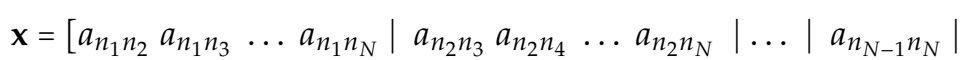

$$
\begin{aligned}
& \left.N_{n_{1}}^{W} N_{n_{2}}^{W} \ldots N_{n_{N}}^{W} \mid N_{n_{1}}^{P V} N_{n_{2}}^{P V} \ldots N_{n_{N}}^{P V}\right]
\end{aligned}
$$

\subsection{Overall Optimization Procedure}

The multi-objective formulation of the problem at hand consists of finding the optimal MG's topology, $\mathcal{S}$, together with the optimal siting for the renewable generation, $\mathcal{T}$. This information is encoded in each individual/coral considered in the Mo-SL-CRO (Section 3.3). The optimization algorithm (Sections 3.1 and 3.2) will then obtain a family of non-dominated solutions $\mathbf{x}$ (Pareto front) in terms of the two health functions considered: $f_{1}(\mathbf{x})=C(\mathbf{x})$ and $f_{2}(\mathbf{x})=E(\mathbf{x})$ that optimize $\mathcal{S}$ and $\mathcal{T}$ (Section 2). The flowchart of the overall optimization procedure is shown in Figure 1. 


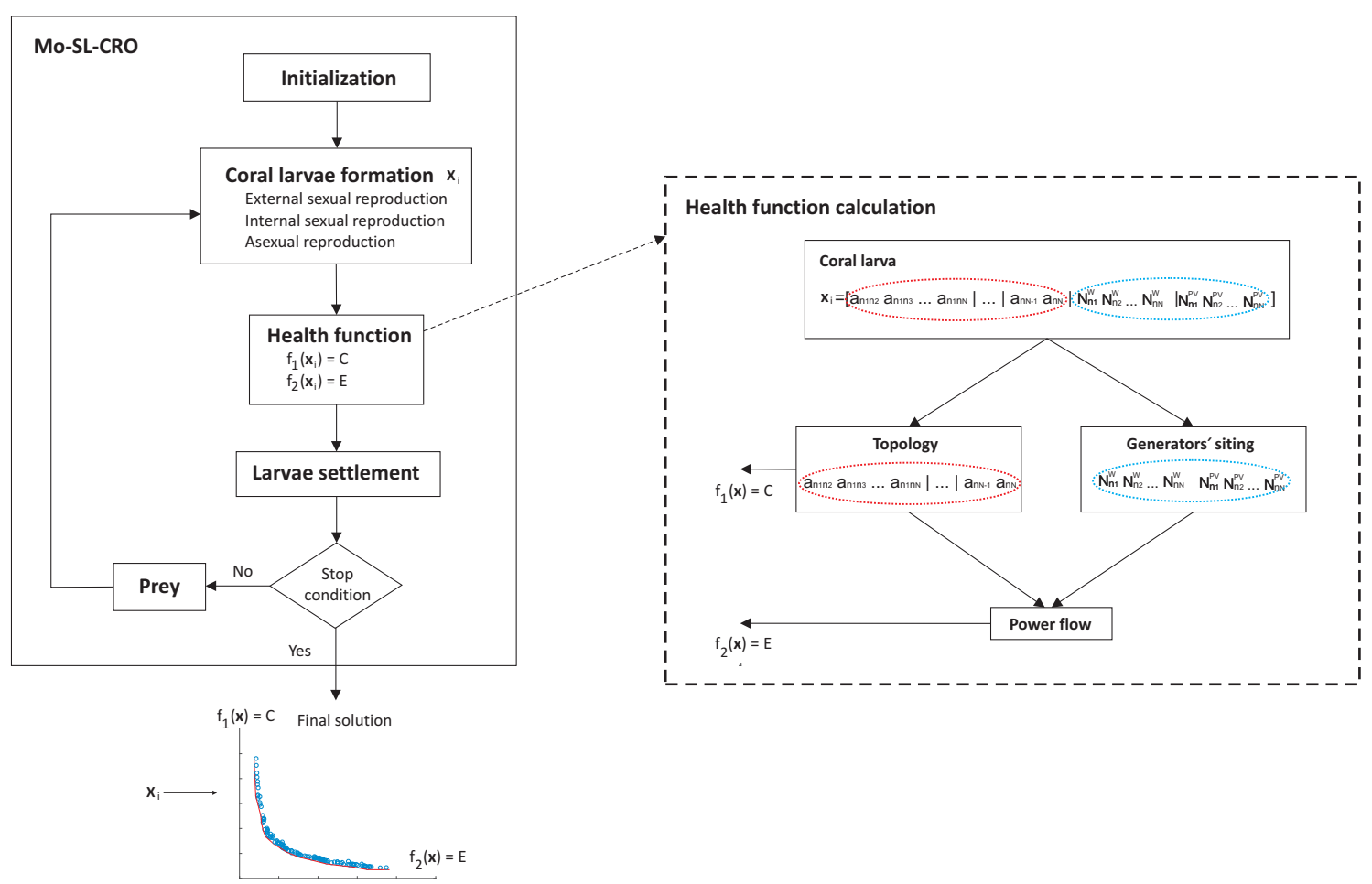

Figure 1. Flowchart of the overall optimization procedure.

\subsection{Multi-Objective Optimization Performance Metrics}

The multi-objective optimization algorithms aim to find a set of non-dominated Pareto optimal solutions. Each of these solutions is not better or worse than the others contained in the front, and it is the decision maker the one that has to choose the solution that suits his/her interests best $[45,46]$. Therefore, when comparing Pareto fronts obtained by different methodologies it is necessary to apply metrics [45-48] that indicate the overall performance of each front. These types of metrics are divided in those in which the optimal front or ideal trade-off is known, or those where an ideal front or function to verify the overall performance of the set of solutions is not needed. The latter are the ones that are used in this work.

These evaluation metrics usually involve three indexes, grouped in two goals:

1. Convergence or closeness: the best solutions (non-dominated) are those more approximate to the Pareto optimal solutions.

2. Diversity: The diversity concept can be separated in two different measures, which in the ideal case should be jointly fulfilled.

- $\quad$ Spread: the best solutions should cover most of the Pareto optimal frontier.

- Spacing: the best solutions should be uniformly distributed (relative distance among solutions) along the Pareto optimal frontier.

These metrics are partly conflicting, therefore there is no metric able to determine the performance of a multi-objective algorithm in an absolute sense. Therefore, there is a clear need of considering at least two performance metrics for adequately assess both goals.

In some cases, the result obtained using the evaluation metrics, depends on the scale and/or intervals of values that each of the objectives to optimize can reach $[45,48]$. For this purpose we have chosen to normalize each non-dominated solution, so that, problems of magnitude, scale, and maximum and minimum values that can take each of the solutions are avoided. Specifically, 
on each Pareto front $Q$, and for each objective $j$, each solution $i$ is transformed into a solution $i^{\prime}$, normalized to $[0,1]$ and therefore maximized for the comparison procedure:

$$
i_{j}^{\prime}=\frac{1}{1+i_{j}}, \forall i \in Q, j=1,2
$$

The metrics most used in the literature and utilized in this work are the following:

- Hypervolume or Hyperarea (HA): This metric calculates the volume in the objective space covered by the solutions of a Pareto front. Mathematically, for each solution $i$ belonging to $Q$, a hypercube $v_{i}$ is constructed with a reference point $W$, in this work $W=(0,0)$. $H A$ takes into consideration both closeness and diversity, providing a combined qualitative measure. The objective of this metric is its maximization in the interval $[0,1]$, being 1 the best possible $H A$.

Note that if normalization is not carried out, this metric would clearly manifest important problems, as the first objective function takes values an order of magnitude more than that of the second objective. A unit improvement in the first objective would reduce $H A$ much more than a unit in the second objective.

- Zitzler's Spread (SPR): SPR measures the spread of the trade-off surface [49]. It is a measure that obtains the length of the diagonal of a hyperbox formed by the extreme function values of the trade-off surface, using the sum of the greatest distance for each objective $i$.

For two objectives, this measure refers to the two solutions with the longest Euclidean distance in the objective space. Thus, the objective of this metric is its maximization, but it is important to note that it does not reveal the distribution of intermediate solutions, becoming paramount its use along with another metric that measures the uniformity of the Pareto front.

- Schott's Spacing (SPA): This metric is calculated with a relative distance measure $d_{i}$ for each solution $i$ on the trade-off [50]. Specifically, $d_{i}$ is the minimum value of the sum of the absolute difference for each objective between the $i$-th solution and any other solution in the obtained non-dominated set (note that it is different from the minimum Euclidean distance). Taking this into account, the standard deviation of the distances $d_{i}$ is calculated, so that, if the solutions are uniformly spaced, the corresponding distance measure will be small. Thus, the objective of this metric is its minimization.

- Hole Relative Size (HRS): The spacing metric SPA computes a mean error with respect to an ideal spacing, but it could have a tendency to hide some important gaps. HRS allows to measure the size of the biggest hole on the trade-off surface between neighbors [48]. Specifically, the distance of each solution with respect to its nearest neighbor is calculated, and then, the maximum of those distances is divided by the mean of the distances of each solution respect to its nearest neighbor. The objective of this metric is its minimization (the best and lowest possible value is 1 , that is, the biggest distance between neighbors coincides with the mean).

\section{Results}

This section describes the MG used in this work: loads' profiles considered, DG and types of generators used, distances separating the nodes in the grid, etc. Later, an assessment of the proposed Mo-SL-CRO algorithm is presented, providing results obtained with different combinations of substrate layers and comparing them with the outcomes of well-known multi-objective algorithms (NSGA-II, Multi-objective Harmony Search).

\subsection{Description of the MG and the Generators and Loads Used}

The proposed multi-objective algorithm has been tested on a twelve-noded MG $(N=12)$ where the nodes are separated by distances shown in Table 1. Eleven different loads (relating to commercial and industrial consumptions), $\mathcal{L}^{i}$, modeled by their real-life annual hourly-consumption profiles, have been used and placed as shown in Figure 2. 
Table 1. Distance separating each pair of nodes in the MG. A distance of 1 corresponds to $100 \mathrm{~m}$. Distances' matrix is symmetrical $l_{i k}=l_{k i}$ (it has not been represented that way for clarity purposes) and all elements in the main diagonal are equal to zero.

\begin{tabular}{cccccccccccc}
\hline Node \# & $\mathbf{2}$ & $\mathbf{3}$ & $\mathbf{4}$ & $\mathbf{5}$ & $\mathbf{6}$ & $\mathbf{7}$ & $\mathbf{8}$ & $\mathbf{9}$ & $\mathbf{1 0}$ & $\mathbf{1 1}$ & $\mathbf{1 2}$ \\
\hline 1 & 0.6 & 0.8 & 1.6 & 1.3 & 0.6 & 1.3 & 0.4 & 1.2 & 1.3 & 2.0 & 1.9 \\
2 & - & 0.5 & 1.1 & 0.7 & 0.3 & 0.8 & 0.6 & 1.0 & 0.9 & 1.4 & 1.4 \\
3 & - & - & 0.7 & 0.6 & 0.8 & 0.9 & 1.0 & 1.5 & 1.2 & 1.4 & 1.2 \\
4 & - & - & - & 0.4 & 1.4 & 0.9 & 1.7 & 1.9 & 1.5 & 1.0 & 0.6 \\
5 & - & - & - & - & 0.9 & 0.5 & 1.3 & 1.4 & 1.0 & 0.9 & 0.6 \\
6 & - & - & - & - & - & 0.7 & 0.4 & 0.7 & 0.7 & 1.5 & 1.5 \\
7 & - & - & - & - & - & - & 1.2 & 1.0 & 0.5 & 0.7 & 0.9 \\
8 & - & - & - & - & - & - & - & 0.8 & 1.0 & 1.9 & 1.9 \\
9 & - & - & - & - & - & - & - & - & 0.4 & 1.6 & 1.8 \\
10 & - & - & - & - & - & - & - & - & - & 1.1 & 1.3 \\
11 & - & - & - & - & - & - & - & - & - & - & 0.5 \\
\hline
\end{tabular}
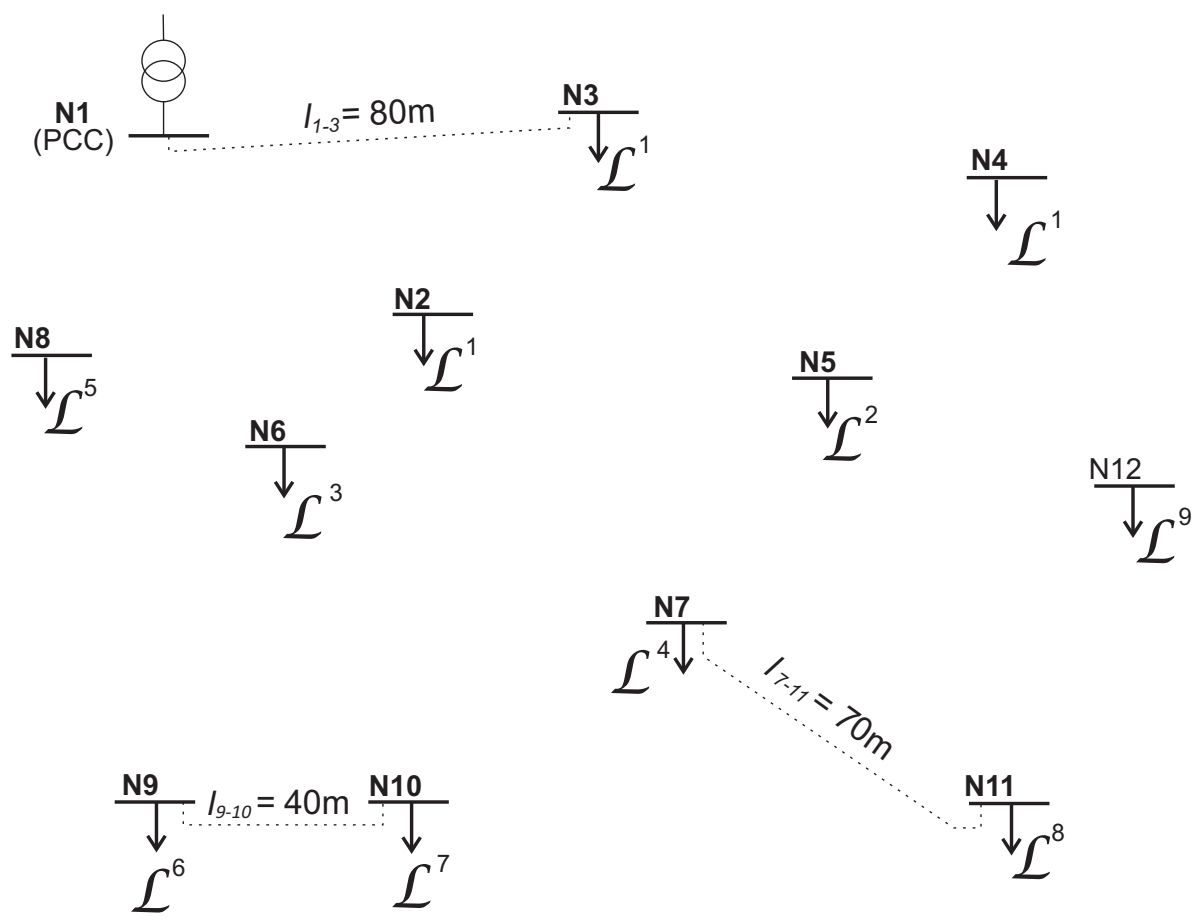

Figure 2. Distribution of the loads in the MG.

The objective is to optimally allocate $800 \mathrm{~kW}$ of photovoltaic generation (using sixteen $50 \mathrm{~kW}$ PV units that add up a total energy generated of 1,316,998.96 kWh) and four $175 \mathrm{~kW}$ micro-wind turbines $(1,302,277.92 \mathrm{kWh}$ generated). At the same time, optimal cross-sectional areas of the lines that should be installed in the MG to minimize energy losses and total cost are determined. According to the normalized cross-sectional areas referred in the IEC 60364-5-52:2009/Corr:2011 (Low-voltage electrical installations) [51], the set of possible line CSAs considered in this work is: $a_{i k} \in\{6,10,16,25,35,50,70,95,120,150,185,240,300,400,500,630\}\left[\mathrm{mm}^{2}\right]$.

To continue characterizing the loads and generators, Table 2 shows the annual micro-wind and photovoltaic energy generation per year, as well as the annual energy consumption of the loads. Later, the annual energy consumption in the microgrid $\left(\sum \mathcal{L}-\sum \mathcal{G}\right)$ divided by the number of nodes is presented. 
Table 2. Annual energy consumption in the MG divided by the number of nodes at the scenario.

\begin{tabular}{cccc}
\hline \multirow{2}{*}{ Profile } & Number of Generators/Loads & \multicolumn{2}{c}{ Energy Consumption [kWh] } \\
\cline { 2 - 4 } & & Per Unit & Total \\
\hline $\mathcal{G}^{P V}$ & 16 & $82,312.00$ & $1,316,992.00$ \\
$\mathcal{G}^{W}$ & 4 & $325,569.00$ & $1,302,276.00$ \\
$\mathcal{L}^{1}$ & 3 & $382,194.42$ & $1,146,583.26$ \\
$\mathcal{L}^{2}$ & 1 & $461,280.58$ & $461,280.58$ \\
$\mathcal{L}^{3}$ & 1 & $445,640.19$ & $445,640.19$ \\
$\mathcal{L}^{4}$ & 1 & $398,193.68$ & $398,193.68$ \\
$\mathcal{L}^{5}$ & 1 & $421,681.87$ & $421,681.87$ \\
$\mathcal{L}^{6}$ & 1 & $400,714.81$ & $400,714.81$ \\
$\mathcal{L}^{7}$ & 1 & $428,316.70$ & $428,316.70$ \\
$\mathcal{L}^{8}$ & 1 & $324,092.84$ & $324,092.84$ \\
$\mathcal{L}^{9}$ & 1 & $323,307.95$ & $323,307.95$ \\
\hline Annual energy consumption divided by $N=12[\mathrm{kWh}]$ & & $\mathbf{1 4 6 , 3 2 2 . 2 1}$ \\
\hline
\end{tabular}

Considering the loads' power consumption at each node $i$ during a time interval $T$ as $\mathcal{L}^{i}=$ $\left\{\mathcal{L}_{(t=1)}^{i}, \mathcal{L}_{(t=2)}^{i}, \ldots, \mathcal{L}_{(t=T)}^{i}\right\}$, and the power generated at each node $i$ during a time interval $T$ as $\mathcal{G}^{i}=$ $\left\{\mathcal{G}_{(t=1)}^{i}, \mathcal{G}_{(t=2)}^{i}, \ldots, \mathcal{G}_{(t=T)}^{i}\right\}$, the DC power flow can be solved [34] obtaining the power flowing through each line is obtained, therefore all lines energy losses may be determined afterwards.

\subsection{Assessment of the Mo-SL-CRO}

First of all, an assessment of the proposed multi-objective substrate-based algorithm has been performed using the tuned parameters showed in Table 3 and randomized initialization. The two health functions considered are those defined by the two conflicting objectives presented in Equations (3) and (4).

Table 3. Mo-SL-CRO optimization parameters.

\begin{tabular}{ll}
\hline Phase & Parameter \\
\hline \multirow{2}{*}{ Inicialization } & Reef size $=14 \times 10$ (140 elements) \\
& $\begin{array}{l}\mu \in\{1 \ldots 7\} \text { (7 possible substrates) } \\
\rho_{0}=0.8 \text { (total of } 112 \text { corals) }\end{array}$ \\
\hline External sexual reproduction & $F_{e}=0.97$ \\
\hline \multirow{2}{*}{ Internal sexual reproduction } & $F_{i}=1-F e=0.03$ \\
& $P_{i}=0.30$ \\
\hline \multirow{2}{*}{ Asexual reproduction } & $F_{a}=0.05$ \\
& $P_{a}=0.005$ \\
\hline \multirow{2}{*}{ Larvae setting } & $\mathcal{N}_{a t t}=3$ \\
\hline \multirow{2}{*}{ Depredation } & Identical corals are not allowed in the reef. \\
\hline Stop criteria & $P_{p}=0.10$ \\
& $F_{p}=0.40$ \\
\hline
\end{tabular}

For this purpose, several experiments have been run considering the reef formed by just one type of substrate at a time. Therefore, seven Pareto fronts have been obtained using the following substrates: (1) Harmony Search (HS), (2) Differential Evolution (DE), (3) 2-points crossover (2PX), (4) Gaussian Mutation (GM), (5) Strange attractors-based mutation (SABM), (6) Polinomial mutation (PM), and (7) 
Simulated Binary Crossover (SBX), and have been compared with the proposed algorithm using all seven substrates in contend (Mo-7SL-CRO). Results obtained are presented in Figure 3 and Table 4.

According to this, the multi-objective Mo-1SL-CRO using the SBX (Simulated Binary Crossover) substrate layer is the best algorithm, as it covers the most hypervolume, $H A$, and has the smallest spacing. The main issue of this algorithm is that it has a very small diversity $(S P R)$. The second best algorithm is the Mo-7SL-CRO, that presents the second best $H A$ and the best SPR, although showing bad spacing between solutions.

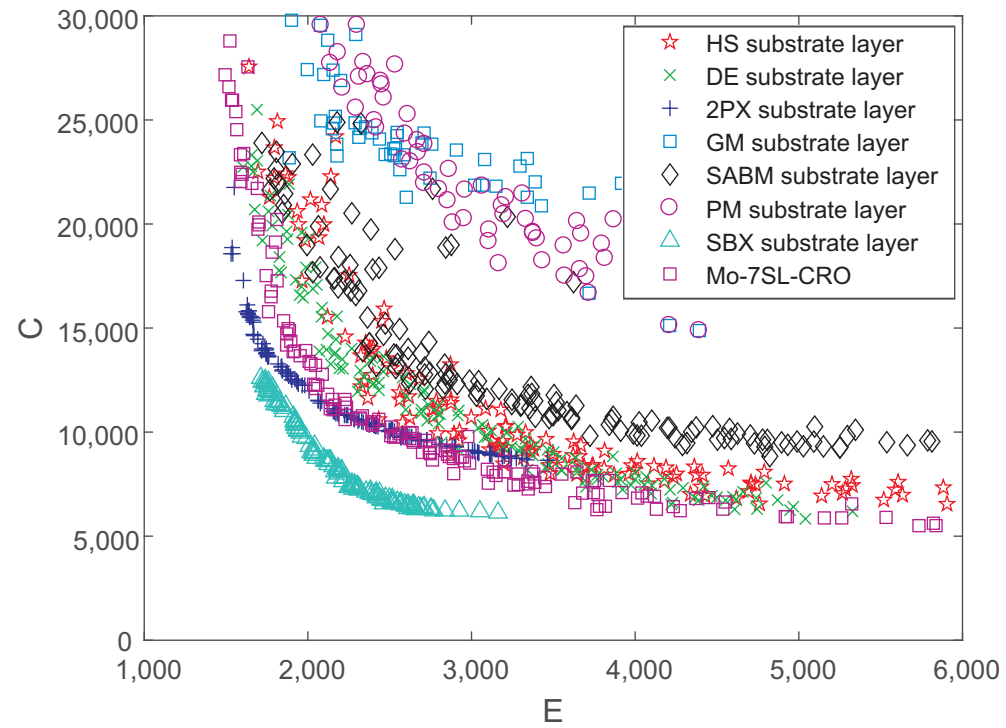

Figure 3. Comparison of the Pareto fronts obtained using multi-objective CRO with the reef formed only by one type of substrate and the Mo-7SL-CRO.

Table 4. Main performance metrics' results corresponding to Figure 3. $\uparrow$ represents Maximization and $\downarrow$ Minimization. The best result for each metric is shown in boldface, while the second one is presented in italics.

\begin{tabular}{|c|c|c|c|c|}
\hline \multirow{2}{*}{ Algorithm } & \multicolumn{4}{|c|}{ Metrics } \\
\hline & $H A(\uparrow)$ & SPR ( $)$ & $S P A(\downarrow)$ & $H R S(\downarrow)$ \\
\hline Mo-1SL-CRO: HS & $6.6817 \times 10^{-8}$ & $4.6625 \times 10^{-4}$ & $8.4446 \times 10^{-6}$ & 1.8472 \\
\hline Mo-1SL-CRO: DE & $7.2183 \times 10^{-8}$ & $4.4331 \times 10^{-4}$ & $6.3684 \times 10^{-6}$ & 2.1950 \\
\hline Mo-1SL-CRO: 2PX & $6.5093 \times 10^{-8}$ & $3.6942 \times 10^{-4}$ & $4.6958 \times 10^{-6}$ & 10.7885 \\
\hline Mo-1SL-CRO: GM & $3.3227 \times 10^{-8}$ & $4.2093 \times 10^{-4}$ & $5.2403 \times 10^{-5}$ & 2.8114 \\
\hline Mo-1SL-CRO: SABM & $5.0976 \times 10^{-8}$ & $3.8077 \times 10^{-4}$ & $6.0658 \times 10^{-6}$ & 2.4426 \\
\hline Mo-1SL-CRO: PM & $2.9044 \times 10^{-8}$ & $3.0797 \times 10^{-4}$ & $6.9765 \times 10^{-6}$ & 2.4630 \\
\hline Mo-1SL-CRO: SBX & $8.5343 \times 10^{-8}$ & $2.8038 \times 10^{-4}$ & $2.7530 \times 10^{-6}$ & 3.2094 \\
\hline Mo-7SL-CRO & $8.1182 \times 10^{-8}$ & $5.1537 \times 10^{-4}$ & $7.5753 \times 10^{-6}$ & 3.7524 \\
\hline
\end{tabular}

Considering the different performances of the substrates chosen (some of them presenting poor results), a thorough study of multi-objective $\mu$-substrates CRO (Mo- $\mu$ SL-CRO) was conducted. Therefore, all combinations of $\mu \in\{1 \ldots 6\}$ were run, resulting in best performance when three specific substrates were used together, namely SBX, 2PX and DE (Mo-3SL-CRO(SBX/2PX/DE)).

Figure 4 and Table 5 present a comparison of the best Mo-3SL-CRO and the Mo-7SL-CRO, together with two well-known multi-objective techniques in the literature: the NSGA-II and the Multi-objective Harmony Search (Mo-HS). Note that for clarity purposes, results obtained with all possible combinations of substrate layers tried are not shown in the table nor in the figure. It can be seen that the Mo-3SL-CRO is the algorithm performing best, producing also a more spreaded and less spaced front than the reference algorithms. 


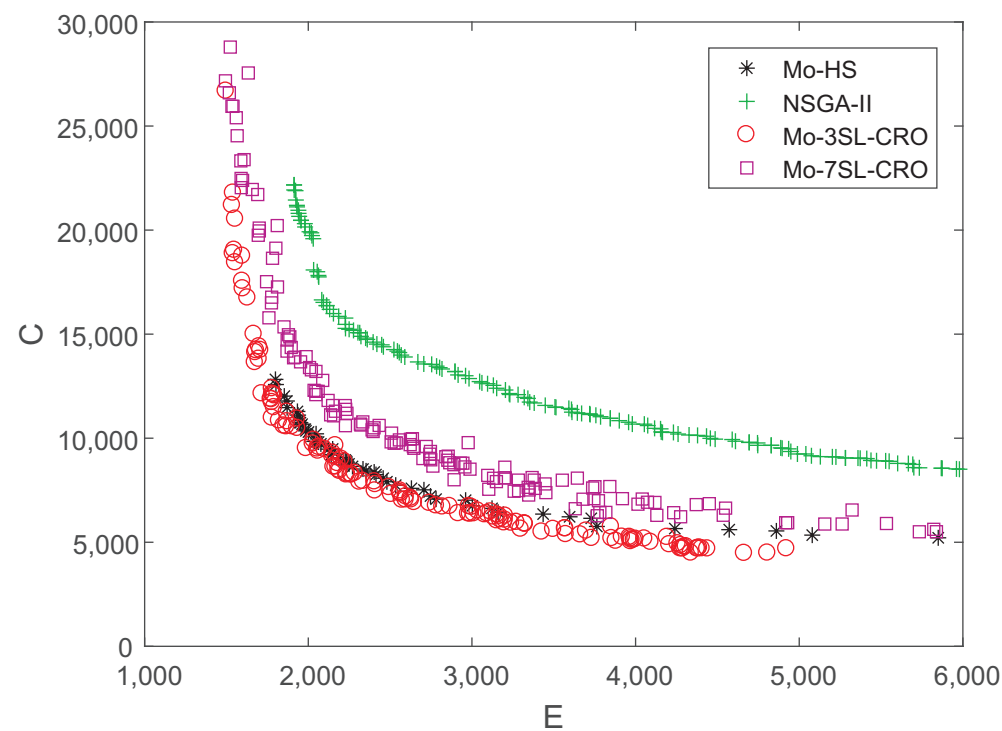

Figure 4. Pareto fronts obtained using Mo-7SL-CRO (multi-objective 7 substrates CRO), Mo-3SL-CRO, multi-objective Harmony Search algorithm (Mo-HS) and NSGA-II.

Table 5. Main performance metrics' results corresponding to Figure 4. $\uparrow$ represents Maximization and $\downarrow$ Minimization. The best result for each metric is shown in boldface, while the second one is presented in italics.

\begin{tabular}{|c|c|c|c|c|}
\hline \multirow{2}{*}{ Algorithm } & \multicolumn{4}{|c|}{ Metrics } \\
\hline & $H A(\uparrow)$ & SPR $(\uparrow)$ & $S P A(\downarrow)$ & $H R S(\downarrow)$ \\
\hline NSGA-II & $4.7811 \times 10^{-8}$ & $3.6453 \times 10^{-4}$ & $1.4696 \times 10^{-6}$ & 3.9872 \\
\hline Mo-HS & $8.6657 \times 10^{-8}$ & $4.3820 \times 10^{-4}$ & $7.9602 \times 10^{-6}$ & 5.9867 \\
\hline Mo-3SL-CRO (SBX/2PX/DE) & $1.0405 \times 10^{-7}$ & $4.8956 \times 10^{-4}$ & $5.7188 \times 10^{-6}$ & 2.9453 \\
\hline Mo-7SL-CRO & $8.1182 \times 10^{-8}$ & $5.1537 \times 10^{-4}$ & $7.5753 \times 10^{-6}$ & 3.7524 \\
\hline
\end{tabular}

Furthermore, a comparison of the best Mo-3SL-CRO (using three substrate layers DE/SBX/2PX) with the Mo-1SL-CRO using only one substrate per experiment was also run and results are shown in Figure 5 and Table 6, along with the multi-objective harmony search algorithm. It can be seen that, if the proposed multi-objective algorithm is run considering only one substrate, performance is not as appropriate as that obtained using all those substrates in contend. Best hyperarea, second best spreading and second best HRS spacing. Again, the Mo-7SL-CRO presents worse performance than the Mo-3SL-CRO, as too many substrate layers in the algorithm introduce noise to the system.

Table 6. Main performance metrics' results corresponding to Figure 5. $\uparrow$ represents Maximization and $\downarrow$ Minimization. The best result for each metric is shown in boldface, while the second one is presented in italics.

\begin{tabular}{|c|c|c|c|c|}
\hline \multirow{2}{*}{ Algorithm } & \multicolumn{4}{|c|}{ Metrics } \\
\hline & $H A(\uparrow)$ & $S P R(\uparrow)$ & $S P A(\downarrow)$ & $H R S(\downarrow)$ \\
\hline Mo-HS & $8.6657 \times 10^{-8}$ & $4.3820 \times 10^{-4}$ & $7.9602 \times 10^{-6}$ & 5.9867 \\
\hline Mo-1SL-CRO: DE & $7.2183 \times 10^{-8}$ & $4.4331 \times 10^{-4}$ & $6.3684 \times 10^{-6}$ & 2.1950 \\
\hline Mo-1SL-CRO: 2PX & $6.5090 \times 10^{-8}$ & $3.6942 \times 10^{-4}$ & $4.7144 \times 10^{-6}$ & 10.5966 \\
\hline Mo-1SL-CRO: SBX & $8.5343 \times 10^{-8}$ & $2.8038 \times 10^{-4}$ & $2.7530 \times 10^{-6}$ & 3.2094 \\
\hline Mo-3SL-CRO (SBX/2PX/DE) & $1.0405 \times 10^{-7}$ & $4.8956 \times 10^{-4}$ & $5.7188 \times 10^{-6}$ & 2.9453 \\
\hline Mo-7SL-CRO & $8.1182 \times 10^{-8}$ & $5.1537 \times 10^{-4}$ & $7.5753 \times 10^{-6}$ & 3.7524 \\
\hline
\end{tabular}




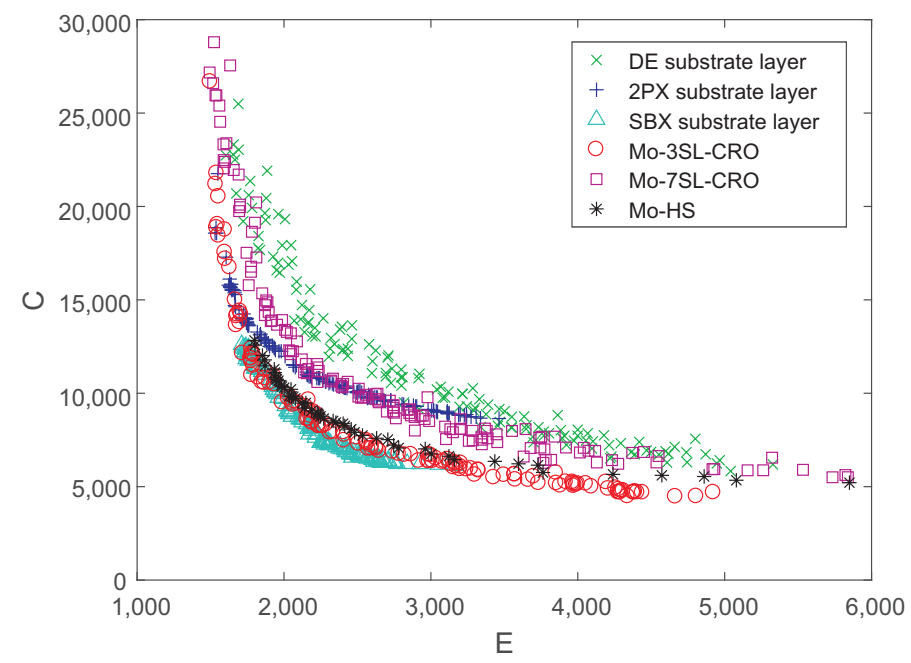

Figure 5. Comparison of the Pareto fronts obtained using multi-objective CRO with the reef formed only by one type of substrate and the Mo-7SL-CRO.

Additionally, a study considering the prey of different fractions of the reef $\left(F_{p}\right)$ as well as different probabilities to perform predation at each iteration $\left(P_{p}\right)$ has also been conducted and can be found in Figure 6 and Table 7. It is important to highlight that prey is needed in the algorithm, as it allows to clear a part of the reef and new solutions (located at other parts of the solutions' space) can enter the reef and form new corals, escaping from local minima and extending the Pareto front. If no prey takes place, it can be observed that the algorithm is able to find good solutions but concentrated on a small area of the solutions' space (only the SPA metric is good, but really poor results in $H A$, SPR and HRS are obtained). On the contrary, high prey probabilities and fractions of corals preyed over-decimate the population and turn the algorithm into a random search, being the frontier to that a $P_{p}=20 \%$ and a $F_{p}=50 \%$. In between, each combination of $P_{p}$ and $F_{p}$ results in more spreaded or less-spaced outcomes. In our case, we chose a prey population of $10 \%$ and a fraction of the reef preyed of $40 \%$, as this combination is a good trade-off between convergence (it presents the second best hyperarea), spreading and spacing of the front (in both cases close to the second best).

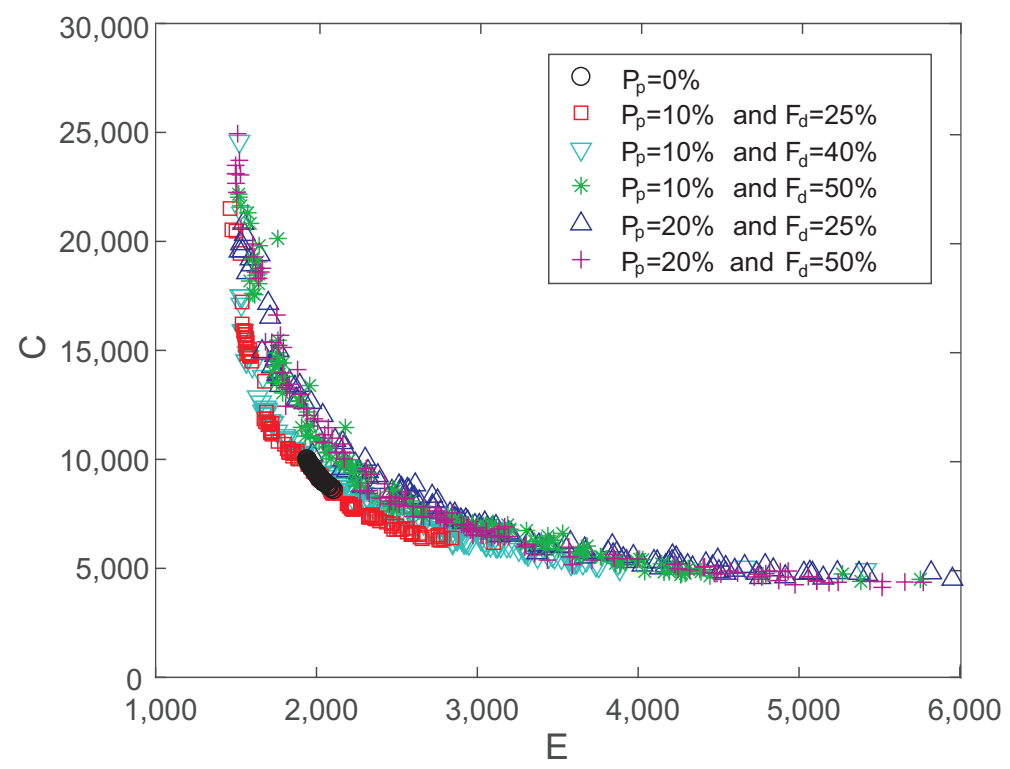

Figure 6. Comparison of the Pareto fronts obtained using the Mo-3SL-CRO (DE/SBCX/2PX) for different prey probabilities $\left(P_{p}\right)$ and fractions of the reef preyed $\left(F_{p}\right)$. 
Table 7. Main performance metrics' results corresponding to the Mo-3SL-CRO presented in Figure 6. $\uparrow$ represents Maximization and $\downarrow$ Minimization. The best result for each metric is shown in boldface, while the second one is presented in italics.

\begin{tabular}{|c|c|c|c|c|}
\hline \multirow{2}{*}{ Algorithm } & \multicolumn{4}{|c|}{ Metrics } \\
\hline & $H A(\uparrow)$ & SPR $(\uparrow)$ & $S P A(\downarrow)$ & HRS $(\downarrow)$ \\
\hline$P_{p}=0 \%$ & $5.9680 \times 10^{-8}$ & $4.3620 \times 10^{-5}$ & $7.2532 \times 10^{-7}$ & 7.2436 \\
\hline$P_{p}=10 \%, F_{p}=25 \%$ & $9.1250 \times 10^{-8}$ & $3.7871 \times 10^{-4}$ & $5.3818 \times 10^{-6}$ & 9.6488 \\
\hline$P_{p}=10 \%, F_{p}=40 \%$ & $1.0405 \times 10^{-7}$ & $4.8956 \times 10^{-4}$ & $5.7188 \times 10^{-6}$ & 2.9453 \\
\hline$P_{p}=10 \%, F_{p}=50 \%$ & $9.9919 \times 10^{-8}$ & $5.0712 \times 10^{-4}$ & $7.4044 \times 10^{-6}$ & 6.5887 \\
\hline$P_{p}=20 \%, F_{p}=25 \%$ & $9.8817 \times 10^{-8}$ & $5.3255 \times 10^{-4}$ & $7.2635 \times 10^{-6}$ & 3.2939 \\
\hline$P_{p}=20 \%, F_{p}=50 \%$ & $1.0412 \times 10^{-7}$ & $5.6316 \times 10^{-4}$ & $7.3454 \times 10^{-6}$ & 4.6866 \\
\hline
\end{tabular}

Figure 7 presents the Pareto front obtained for the best configuration of the Mo- $\mu$ SL-CRO: best three substrates (DE/SBX/2PX) and $P_{p}=10 \%$ and $F_{d}=40 \%$. Table 8 shows the numerical results obtained for three different solutions in the Pareto front, depicted as $\mathcal{X}_{P 1}, \mathcal{X}_{P 2}$, and $\mathcal{X}_{P 3}$. Solution $\mathcal{X}_{P 1}$ is obtained using the max-min approach [29] for the Pareto front and represents the best compromise between the two objective functions considered ( $E$ and $C)$. Solution $\mathcal{X}_{P 2}$ is the one that minimizes the energy losses $(E)$ and Solution $\mathcal{X}_{P 3}$ is the one that minimizes the cost $(C)$.

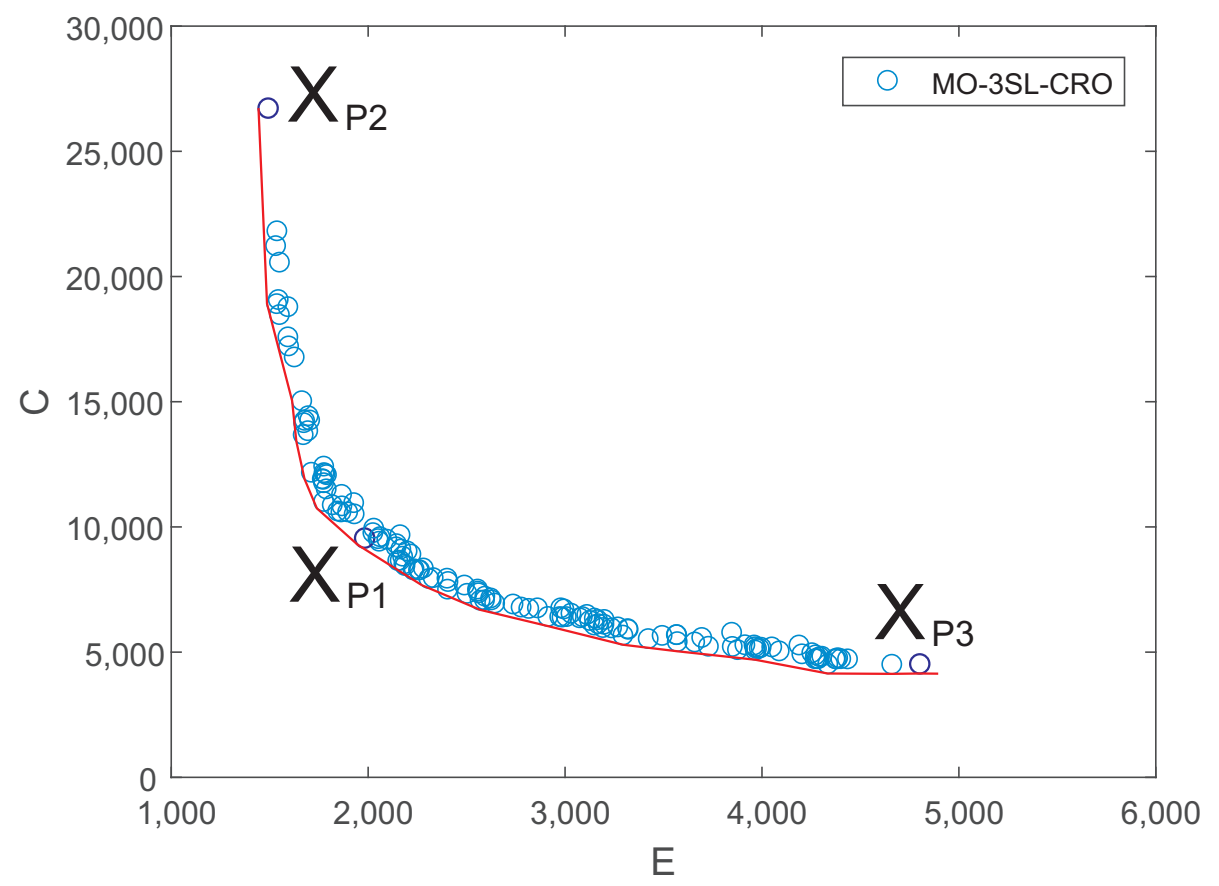

Figure 7. Pareto front for the best Mo-SL-CRO configuration: 3 substrates (DE/SBCX/2PX), $P_{p}=10 \%$ and $F_{d}=40 \%$. Points $\mathcal{X}_{P 1}, \mathcal{X}_{P 2}$, and $\mathcal{X}_{P 3}$ represent three solutions chosen whose results are presented in Table 8.

Table 8. MG lines' energy losses, $E$, and their cost, $C$, using the Mo-3SL-CRO.

\begin{tabular}{ccc}
\hline Point & Energy Losses & Cost \\
\hline $\mathcal{X}_{P 1}$ & 1988.30 & 9506.90 \\
$\mathcal{X}_{P 2}$ & 1498.00 & $26,680.00$ \\
$\mathcal{X}_{P 3}$ & 4807.00 & 4483.00 \\
\hline
\end{tabular}


Table 9 presents a comparison of the results obtained with the proposed algorithm and the well-known NSGA-II algorithm for the best compromise solution $\mathcal{X}_{P 1}$ at each Pareto (using the max-min approach). A simultaneous reduction of the energy losses in a $23 \%$ and the costs in a $31 \%$ reinforce the goodness of the Mo-SL-CRO.

Table 9. Comparison of the MG lines' cost, $C$, and their energy losses, $E$, using the Mo-3SL-CRO and the NSGA-II algorithm for the best compromise solution $\mathcal{X}_{P 1}$ at each Pareto.

\begin{tabular}{ccccc}
\hline \multirow{2}{*}{ Optimization Algorithm } & \multicolumn{2}{c}{ Energy Losses } & \multicolumn{2}{c}{ Cost } \\
\cline { 2 - 5 } & $\boldsymbol{E}[\mathbf{k W h}]$ & Improvement (\%) & $\boldsymbol{C}$ [Monetary Units] & Improvement (\%) \\
\hline NSGA-II & 2589.00 & - & $13,903.00$ & - \\
Mo-3SL-CRO & 1988.30 & $23.20 \%$ & 9506.90 & $31.62 \%$ \\
\hline
\end{tabular}

Table 10 presents the optimal topology matrix $\mathcal{S}$ associated to solutions $\mathcal{X}_{P 1}, \mathcal{X}_{P 2}$ and $\mathcal{X}_{P 3}$. It can be seen that the compromise solution $\left(\mathcal{X}_{P 1}\right)$ is a star (radial) network where the central node is the PCC and is also equipped with several extra lines that connect the secundary nodes to other close ones. The expensive solution $\left(\mathcal{X}_{P 2}\right)$ is a mesh network, almost fully connected, with high cross-sectional area lines, resulting in low energy losses. Finally, the cheap solution $\left(\mathcal{X}_{P 3}\right)$ has few high-CSA lines that are needed to let the power generated/consumed at the nodes flow, and few other lines to guarantee that each node is connected, at least, to one node (a restriction set to the problem).

Table 10. Optimal topology matrix $\mathcal{S}$ for solutions $\mathcal{X}_{P 1}, \mathcal{X}_{P 2}$ and $\mathcal{X}_{P 3}$. Element $\mathcal{S}(i, k)$ represents the cross-sectional area of the line connecting nodes $i$ and $k$. Elements $a_{i k}=0$ represent that there is no line connecting those nodes. Note that matrix $\mathcal{S}$ is symmetrical, as $a_{i k}=a_{k i}$, and elements $a_{i i}=0$, as none of the nodes are connected to itself.

\begin{tabular}{cccccccccccccc}
\hline Solution & Node \# & $\mathbf{1}$ & $\mathbf{2}$ & $\mathbf{3}$ & $\mathbf{4}$ & $\mathbf{5}$ & $\mathbf{6}$ & $\mathbf{7}$ & $\mathbf{8}$ & $\mathbf{B}$ & $\mathbf{1 0}$ & $\mathbf{B}$ & $\mathbf{1 2}$ \\
\hline \multirow{6}{*}{$\mathcal{X}_{P 1}$} & 1 & 0 & 500 & 500 & 500 & 500 & 500 & 500 & 500 & 500 & 500 & 500 & 0 \\
& 2 & 500 & 0 & 185 & 0 & 240 & 500 & 0 & 0 & 0 & 16 & 0 & 0 \\
& 3 & 500 & 185 & 0 & 0 & 0 & 0 & 0 & 0 & 0 & 0 & 0 & 35 \\
& 4 & 500 & 0 & 0 & 0 & 500 & 0 & 0 & 0 & 0 & 0 & 0 & 300 \\
& 5 & 500 & 240 & 0 & 500 & 0 & 0 & 0 & 0 & 0 & 0 & 0 & 0 \\
& 6 & 500 & 500 & 0 & 0 & 0 & 0 & 0 & 500 & 0 & 300 & 0 & 0 \\
& 7 & 500 & 0 & 0 & 0 & 0 & 0 & 0 & 0 & 0 & 0 & 500 & 0 \\
& 8 & 500 & 0 & 0 & 0 & 0 & 500 & 0 & 0 & 0 & 10 & 0 & 0 \\
& 9 & 500 & 0 & 0 & 0 & 0 & 0 & 0 & 0 & 0 & 500 & 0 & 0 \\
& 10 & 500 & 16 & 0 & 0 & 0 & 300 & 0 & 10 & 500 & 0 & 0 & 0 \\
& 11 & 500 & 0 & 0 & 0 & 0 & 0 & 500 & 0 & 0 & 0 & 0 & 400 \\
& 12 & 0 & 0 & 35 & 300 & 0 & 0 & 0 & 0 & 0 & 0 & 400 & 0 \\
\hline \multirow{6}{*}{$\mathcal{X}_{P 2}$} & 1 & 0 & 500 & 500 & 500 & 500 & 500 & 500 & 500 & 500 & 500 & 500 & 500 \\
& 2 & 500 & 0 & 95 & 6 & 0 & 400 & 0 & 500 & 50 & 0 & 120 & 400 \\
& 3 & 500 & 95 & 0 & 10 & 500 & 0 & 0 & 500 & 500 & 500 & 500 & 500 \\
& 4 & 500 & 6 & 10 & 0 & 500 & 500 & 500 & 0 & 0 & 500 & 0 & 500 \\
& 5 & 500 & 0 & 500 & 500 & 0 & 500 & 500 & 0 & 150 & 500 & 500 & 500 \\
& 6 & 500 & 400 & 0 & 500 & 500 & 0 & 300 & 500 & 0 & 0 & 0 & 70 \\
& 7 & 500 & 0 & 0 & 500 & 500 & 300 & 0 & 120 & 500 & 500 & 500 & 0 \\
& 8 & 500 & 500 & 500 & 0 & 0 & 500 & 120 & 0 & 240 & 500 & 10 & 240 \\
& 9 & 500 & 50 & 500 & 0 & 150 & 0 & 500 & 240 & 0 & 400 & 500 & 500 \\
& 10 & 500 & 0 & 500 & 500 & 500 & 0 & 500 & 500 & 400 & 0 & 0 & 500 \\
& 11 & 500 & 120 & 500 & 0 & 500 & 0 & 500 & 10 & 500 & 0 & 0 & 120 \\
& 12 & 500 & 400 & 500 & 500 & 500 & 70 & 0 & 240 & 500 & 500 & 120 & 0 \\
\hline
\end{tabular}


Table 10. Cont.

\begin{tabular}{cccccccccccccc}
\hline Solution & Node \# & $\mathbf{1}$ & $\mathbf{2}$ & $\mathbf{3}$ & $\mathbf{4}$ & $\mathbf{5}$ & $\mathbf{6}$ & $\mathbf{7}$ & $\mathbf{8}$ & $\mathbf{B}$ & $\mathbf{1 0}$ & $\mathbf{B}$ & $\mathbf{1 2}$ \\
\hline \multirow{6}{*}{} & 1 & 0 & 500 & 500 & 185 & 0 & 500 & 240 & 500 & 0 & 0 & 0 & 0 \\
& 2 & 500 & 0 & 16 & 0 & 500 & 500 & 0 & 0 & 0 & 0 & 0 & 0 \\
& 3 & 500 & 16 & 0 & 0 & 0 & 0 & 0 & 0 & 0 & 0 & 0 & 0 \\
& 4 & 185 & 0 & 0 & 0 & 500 & 0 & 0 & 0 & 0 & 0 & 0 & 300 \\
& 5 & 0 & 500 & 0 & 500 & 0 & 0 & 0 & 0 & 0 & 0 & 0 & 0 \\
& 6 & 500 & 500 & 0 & 0 & 0 & 0 & 0 & 25 & 0 & 500 & 0 & 0 \\
& 7 & 240 & 0 & 0 & 0 & 0 & 0 & 0 & 0 & 0 & 0 & 500 & 0 \\
& 8 & 500 & 0 & 0 & 0 & 0 & 25 & 0 & 0 & 0 & 0 & 0 & 0 \\
& 9 & 0 & 0 & 0 & 0 & 0 & 0 & 0 & 0 & 0 & 500 & 0 & 0 \\
& 10 & 0 & 0 & 0 & 0 & 0 & 500 & 0 & 0 & 500 & 0 & 0 & 0 \\
& 11 & 0 & 0 & 0 & 0 & 0 & 0 & 500 & 0 & 0 & 0 & 0 & 400 \\
& 12 & 0 & 0 & 0 & 300 & 0 & 0 & 0 & 0 & 0 & 0 & 400 & 0 \\
\hline
\end{tabular}

Table 11 presents the optimal number of generators allocated at each node (matrix $\mathcal{T}$ ) by the Mo-3SL-CRO algorithm for solutions $\mathcal{X}_{P 1}, \mathcal{X}_{P 2}$ and $\mathcal{X}_{P 3}$. As expected, in all solutions, generators are distributed around all nodes, presenting more generation close to more consuming loads (bear in mind that there is one load connected to each node as depicted in Figure 2). Furthermore, in the moderate and low costs solutions, it can be observed that the more generation produced in one node, the more lines connecting that node to closer nodes to distribute the power generated to them.

Table 11. Optimal number of wind turbines $\left(N^{W}\right)$ and PV generators $\left(N^{P V}\right)$ allocated for solution $\mathcal{X}_{P 1}$. The number of micro-wind turbines and photovoltaic generators at each node is provided. Note that Node \#1 (the PCC) is not presented in the table, as a restriction applies and no generation can be allocated.

\begin{tabular}{ccccccc}
\hline & \multicolumn{2}{c}{$\mathcal{X}_{P 1}$} & \multicolumn{2}{c}{$\mathcal{X}_{P 2}$} & \multicolumn{2}{c}{$\mathcal{X}_{P 3}$} \\
\hline Node \# & $N^{P V}$ & $N^{W}$ & $N^{P V}$ & $N^{W}$ & $N^{P V}$ & $N^{W}$ \\
\hline 2 & 2 & - & 3 & 1 & 4 & - \\
3 & 1 & 1 & - & - & 1 & - \\
4 & 1 & - & 2 & 1 & - & - \\
5 & - & 1 & 1 & 1 & - & 1 \\
6 & 2 & - & 2 & - & 1 & - \\
7 & 1 & 1 & 1 & - & - & 1 \\
8 & 2 & - & - & - & 2 & 1 \\
9 & 2 & - & 2 & - & 3 & 1 \\
10 & 2 & 1 & 1 & 1 & - & - \\
11 & - & - & 1 & - & 1 & - \\
12 & 3 & - & 3 & - & 4 & - \\
\hline
\end{tabular}

\section{Conclusions}

The Multi-objective Substrate Layers Coral Reefs Optimization algorithm (Mo-SL-CRO), is presented in this work to solve the problem of determining the best Microgrid's topology (lines interconnecting the nodes) and the optimal renewable generation siting in the MG. For this purpose, two conflicting objectives are used: the cost of the infrastructures and their deployment, and the energy losses produced along the lines of the MG. On the one hand, deployment costs will increase as a more interconnected topology is designed (the more nodes connected to each other, the more expensive a topology is). Besides, the lines connecting each pair of nodes can have low cross-sectional areas, resulting in less material and, thus, a cheaper solution, or high CSAs, resulting in higher infrastructures' 
cost. On the other hand, higher CSAs result in lower energy losses in the line. Moreover, change in the CSAs results in a different power flowing along the lines, thus a power redistribution along the different lines considered in the MG.

The Mo-SL-CRO outperforms two well-known multi-objective algorithms, the NSGA-II and the multi-objective Harmony search, and presents an interesting feature: the user can implement as many substrate layers as wanted, or even remove those that display bad performance for the problem and include new substrates. In the abovementioned aim, the combined use of three substrate layers fits best in the optimization process, being these substrates a layer based on Differential Evolution, another one based on a two point crossover and, finally, a Simulated Binary crossover to obtain new candidates for the following iteration. Each substrate layer on its own does not produce a result as good as that achieved with all of them jointly, and each substrate layer contributes with a special feature: some improve the results, others stretch out the Pareto optimal-fronts, some perform best in certain areas of the search space while others on different ones, etc. For this reason, the novel Mo-SL-CRO comes out as an interesting optimization technique.

Regarding the field of application, the proposed algorithm shows important results in the joint optimization of the MG's topology and the generators placement, suggesting that this technique may help decision-makers to analyze solutions and determine best strategies based on the multi-objective options. A comparison between the proposed Mo-3SL-CRO algorithm and a fair reference as the NSGA-II algorithm shows, for the best compromise solution at each Pareto front, a cost improvement of $31 \%$ and, simultaneously, an energy losses reduction of $23 \%$, confirming the good results of the proposed algorithm.

Author Contributions: Conceptualization, S.J.-F., J.D.S. and S.S.-S.; Methodology, S.J.-F., J.D.S., A.P.-F. and S.S.-S.; Software, C.C.-G. and R.M.-P.; Data curation, J.C.F.; Formal analysis, S.J.-F. and S.S.-S.; Project administration, A.P.-F.; Supervision, S.S.-S.; Writing-original draft preparation, S.J.-F.; writing—review and editing, R.M.-P. and S.S.-S.

Funding: This research was partially funded by Ministerio de Economía, Industria y Competitividad, project number TIN2017-85887-C2-1-P and TIN2017-85887-C2-2-P, and by the Comunidad Autónoma de Madrid, project number S2013ICE-2933_02.

Conflicts of Interest: The authors declare no conflict of interest.

\section{Nomenclature}

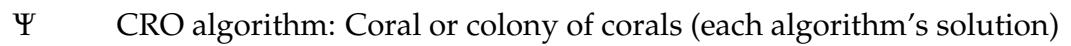

$a_{i k} \quad$ Cross-sectional area of the line connecting nodes $i$ and $k$

C Line's deployment cost

$C_{\text {ins }} \quad$ Line's installation unity cost per $\mathrm{m}$

$C_{\text {mat }}$ Line's material unity cost per $\mathrm{m} \cdot \mathrm{mm}^{2}$

CSA Cross-sectional area

DG Distributed Generation

E Line's energy losses

$\mathcal{E} \quad$ Energy losses in the line connecting nodes $i$ and $k$

$F_{a} \quad$ CRO algorithm: Fraction of the reef's existing corals that generate new larvae during asexual reproduction

$F_{e} \quad$ CRO algorithm: Fraction of the reef's existing corals that generate new larvae during external sexual reproduction

$F_{i} \quad$ CRO algorithm: Fraction of the reef's existing corals that generate new larvae during internal sexual reproduction

$F_{p} \quad$ CRO algorithm: Fraction of the reef's existing corals that are preyed

$\mathcal{G}$ Renewable generator

$\mathcal{L}_{i k} \quad$ Power losses in the line that connects nodes $i$ and $k$

$l_{i k} \quad$ Length of the line connecting nodes $i$ and $k$

$\mathcal{L}$ Load

$\mu \quad$ Number of possible substrates 


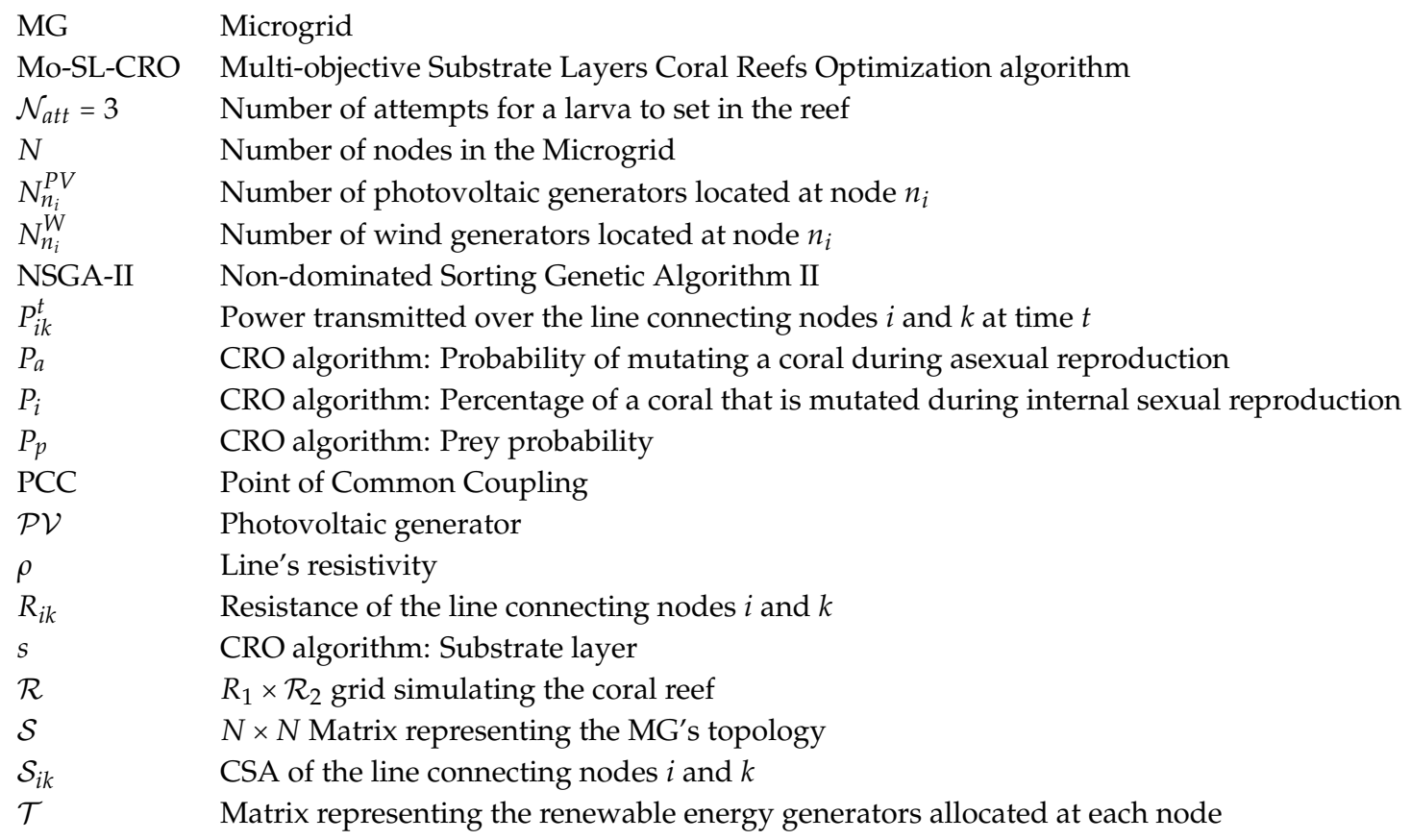

\section{References}

1. Hittinger, E.; Wiley, T.; Kluza, J.; Whitacre, J. Evaluating the value of batteries in microgrid electricity systems using an improved Energy Systems Model. Energy Convers. Manag. 2015, 89, 458-472. [CrossRef]

2. Guerrero, J.; Vásquez, J. Microgrids; Wiley IEEE: Hoboken, NJ, USA, 2016.

3. Wang, R.; Wang, P.; Xiao, G. A robust optimization approach for energy generation scheduling in microgrids. Energy Convers. Manag. 2015, 106, 597-607. [CrossRef]

4. Gamarra, C.; Guerrero, J.M. Computational optimization techniques applied to microgrids planning: A review. Renew. Sustain. Energy Rev. 2015, 48, 413-424. [CrossRef]

5. Keane, A.; Ochoa, L.F.; Borges, C.L.T.; Ault, G.W.; Alarcon-Rodriguez, A.D.; Currie, R.; Pilo, F.; Dent, C.; Harrison, G.P. State-of-the-art techniques and challenges ahead for distributed generation planning and optimization. IEEE Trans. Power Syst. 2013, 28, 1493-1502. [CrossRef]

6. Hossain, E.; Kabalci, E.; Bayindir, R.; Perez, R. Microgrid testbeds around the world: State of art. Energy Convers. Manag. 2014, 86, 132-153. [CrossRef]

7. Chen, C.; Duan, S. Optimal allocation of distributed generation and energy storage system in microgrids. IET Renew. Power Gener. 2014, 8, 581-589. [CrossRef]

8. Le, D.T.; Kashem, M.A.; Negnevitsky, M.; Ledwich, G. Optimal distributed generation parameters for reducing losses with economic consideration. In Proceedings of the 2007 IEEE Power Engineering Society General Meeting, Tampa, FL, USA, 24-28 June 2007; pp. 1-8.

9. Moradi, M.H.; Abedini, M. A novel method for optimal DG units capacity and location in Microgrids. Int. J. Electr. Power Energy Syst. 2016, 75, 236-244. [CrossRef]

10. Gözel, T.; Hocaoglu, M.H. An analytical method for the sizing and siting of distributed generators in radial systems. Electr. Power Syst. Res. 2009, 79, 912-918. [CrossRef]

11. Ghosh, S.; Ghoshal, S.P.; Ghosh, S. Optimal sizing and placement of distributed generation in a network system. Int. J. Electr. Power Energy Syst. 2010, 32, 849-856. [CrossRef]

12. Moradi, M.H.; Abedini, M. A combination of genetic algorithm and particle swarm optimization for optimal DG location and sizing in distribution systems. Int. J. Electr. Power Energy Syst. 2012, 34, 66-74. [CrossRef]

13. Doagou-Mojarrad, H.; Gharehpetian, G.B.; Rastegar, H.; Olamaei, J. Optimal placement and sizing of DG (distributed generation) units in distribution networks by novel hybrid evolutionary algorithm. Energy 2013, 54, 129-138. [CrossRef]

14. Devi, S.; Geethanjali, M. Application of Modified Bacterial Foraging Optimization algorithm for optimal placement and sizing of Distributed Generation. Expert Syst. Appl. 2014, 41, 2772-2781. [CrossRef] 
15. Sheng, W.; Liu, K.Y.; Meng, X.; Li, Y. Optimal placement and sizing of distributed generation via an improved nondominated sorting genetic algorithm II. IEEE Trans. Power Deliv. 2015, 30, 569-578. [CrossRef]

16. Gupta, S.; Saxena, A.; Soni, B.P. Optimal Placement Strategy of Distributed Generators based on Radian Basis Function Neural Network in Distribution Networks. Procedia Comput. Sci. 2015, 57, 249-257. [CrossRef]

17. Obara, S.; Kawai, M.; Kawae, O.; Morizane, Y. Operational planning of an independent microgrid containing tidal power generators, SOFCs, and photovoltaics. Appl. Energy 2013, 102, 1343-1357. [CrossRef]

18. Zhao, B.; Zhang, X.; Li, P.; Wang, K.; Xue, M.; Wang, C. Optimal sizing, operating strategy and operational experience of a stand-alone microgrid on Dongfushan Island. Appl. Energy 2014, 113, 1656-1666. [CrossRef]

19. Cuadra, L.; Salcedo-Sanz, S.; del Ser, J.; Jiménez-Fernández, S.; Geem, Z.W. A critical review of robustness in power grids using complex networks concepts. Energies 2015, 8, 9211-9265. [CrossRef]

20. Wang, Y.; Huang, Y.; Wang, Y.; Li, F.; Zhang, Y.; Tian, C. Operation Optimization in a Smart Micro-Grid in the Presence of Distributed Generation and Demand Response. Sustainability 2018, 10, 847. [CrossRef]

21. Zeng, Z.; Yang, H.; Zhao, R.; Cheng, C. Topologies and control strategies of multi-functional grid-connected inverters for power quality enhancement: A comprehensive review. Renew. Sustain. Energy Rev. 2013, 24, 223-270. [CrossRef]

22. Prabha, D.R.; Jayabarathi, T. Optimal placement and sizing of multiple distributed generating units in distribution networks by invasive weed optimization algorithm. Ain Shams Eng. J. 2016, 7, 683-694. [CrossRef]

23. Mohan, V.J.; Albert, R.A.D. Optimal sizing and sitting of distributed generation using Particle Swarm Optimization Guided Genetic Algorithm. Adv. Comput. Sci. Technol. 2017, 10, 709-720.

24. Nekooei, K.; Farsangi, M.M.; Nezamabadi-Pour, H.; Lee, K.Y. An improved multi-objective harmony search for optimal placement of DGs in Distribution Systems. IEEE Trans. Smart Grid 2013, 4, 557-567. [CrossRef]

25. Rajaram, R.; Kumar, K.S.; Rajasekar, N. Power system reconfiguration in a radial distribution network for reducing losses and to improve voltage profile using modified plant growth simulation algorithm with distributed generation (DG). Energy Rep. 2015, 1, 116-122. [CrossRef]

26. Naveen, S.; Kumar, K.S.; Rajalakshmi, K. Distribution system reconfiguration for loss minimization using modified bacterial foraging optimization algorithm. Electr. Power Energy Syst. 2015, 69, 90-97. [CrossRef]

27. Azad-Farsani, E. Loss minimization in distribution systems based on LMP calculation using honey bee mating optimization and point estimate method. Energy 2017, 140, 1-9. [CrossRef]

28. Yang, Y.; Yang, B.; Niu, M. Adaptive infinite impulse response system identification using opposition based hybrid coral reefs optimization algorithm. Appl. Intell. 2018, 48, 1689-1706. [CrossRef]

29. Haghifam, M.-R.; Falaghi, H.; Malik, O.P. Risk-based distributed generation placement. IET Gerner. Transm. Distrib. 2008, 2, 252-260. [CrossRef]

30. Zhang, H.-J.; Feng, Y.-B.; Lin, K.-P. Application of Multi-Species Differential Evolution Algorithm in Sustainable Microgrid Model. Sustainability 2018, 10, 2694. [CrossRef]

31. Salcedo-Sanz, S.; del Ser, J.; Landa-Torres, I.; Gil-López, S.; Portilla-Figueras, J.A. The Coral Reefs Optimization algorithm: A novel metaheuristic for efficiently solving optimization problems. Sci.World J. 2014, 2014, 739768. [CrossRef] [PubMed]

32. Salcedo-Sanz, S.; Camacho-Gómez, C.; Mallol-Poyato, R.; Jiménez-Fernández, S.; del Ser, J. A novel Coral Reefs Optimization algorithm with substrate layers for optimal battery scheduling optimization in micro-grids. Soft Comput. 2016, 20, 4287-4300. [CrossRef]

33. Kothari, D.P.; Nagrath, I.J. Modern Power System Analysis; Mc Graw-Hill: New York, NY, USA, 2008.

34. Stott, B.; Jardim, J.; Alsac, O. DC power flow revisited. IEEE Trans. Power Syst. 2009, 24, 1290-1300. [CrossRef]

35. Durán-Rosal, A.M.; Gutiérrez, P.A.; Salcedo-Sanz, S.; Hervás-Martínez, C. A statistically-driven Coral Reef Optimization algorithm for optimal size reduction of time series. Appl. Soft Comput. 2018, 63, 139-153. [CrossRef]

36. Salcedo-Sanz, S.; García-Díaz, P.; Portilla-Figueras, J.A.; del Ser, J. Coral Reefs optimization algorithm for optimal mobile network development with electromagnetic pollution control criterion. Appl. Soft Comput. 2014, 24, 239-248. [CrossRef]

37. Salcedo-Sanz, S. A review on the Coral Reefs Optimization algorithm: New developing lines and current applications. Prog. Artif. Intell. 2017, 6, 1-15. [CrossRef]

38. Deb, K.; Pratap, A.; Agarwal, S.; Meyarivan, T. A fast and elitist multiobjective genetic algorithm: NSGA-II. IEEE Trans. Evol. Comput. 2002, 6, 182-197. [CrossRef] 
39. Geem, Z.W.; Kim, J.H.; Loganathan, G.V. A new heuristic optimization algorithm: Harmony search. Simulation 2001, 76, 60-68. [CrossRef]

40. Storn, R.; Price, K. Differential Evolution-A simple and efficient heuristic for Global Optimization over Continuous Spaces. J. Glob. Optim. 1997, 11, 341-359. [CrossRef]

41. Salcedo-Sanz, S. Modern meta-heuristics based on nonlinear physics processes: A review of models and design procedures. Phys. Rep. 2016, 655, 1-70. [CrossRef]

42. Grassberger, P.; Procaccia, I. Characterization of strange attractors. Phys. Rev. Lett. 1983, 50, 346-349. [CrossRef]

43. Deb, K.; Agrawal, R.B. Simulated Binary Crossover for Continuous Search Space. Complex Syst. 1995, 9, 115-148.

44. Deb, K.; Goyal, M. A combined genetic adaptive search (GeneAS) for engineering design. Comput Sci. Inform. 1996, 26, 30-45.

45. Deb, K. Multi-Objective Optimization Using Evolutionary Algorithms; Wiley-Interscience Series in Systems and Optimization; Wiley: Hoboken, NJ, USA, 2004.

46. Cui, Y.; Geng, Z.; Zhu, Q.; Han, Y. Review: Multi-objective optimization methods and application in energy saving. Energy 2017, 125, 681-704. [CrossRef]

47. Yen, G.; Zhenan, H. Performance metrics ensemble for multiobjective evolutionary algorithms. IEEE Trans. Evol. Comput. 2014, 18, 131-144. [CrossRef]

48. Collette, Y.; Patrick, S. Multiobjective Optimization—Principles and Case Studies; Springer: Berlin, Germany, 2004.

49. Zitzler, E. Evolutionary Algorithms for Multiobjective Optimization: Methods and Applications; Springer: Berlin, Germany, 1999.

50. Schott, J.R. Fault tolerant design using single and multicriteria genetic algorithms optimization. Master's Thesis, Department of Aeronautics and Astronautics, Massachusetts Institute of Technology, Cambridge, MA, USA, 1995.

51. International Standard IEC 60364-5-52:2009/Corr:2011; Corrigendum 2011; International Electrotechnical Commision: Genève, Switzerland, 2009. 